\title{
EDITORIAL
}

\section{Current and Emerging Therapies for Adrenocortical Carcinoma - Review}

Department of Endocrinology, Diabetology and Isotope Therapy, Wroclaw Medical University, Poland

A - research concept and design; $\mathbf{B}$ - collection and/or assembly of data; $\mathbf{C}$ - data analysis and interpretation;

$\mathbf{D}$ - writing the article; $\mathbf{E}$ - critical revision of the article; $\mathbf{F}$ - final approval of article; $\mathbf{G}$ - other

\begin{abstract}
Adrenocortical carcinoma (ACC) is a rare malignancy with poor prognosis. Patients may present with hormone excess or a local mass effect. The most common imaging techniques (CT and MRI) use both size and appearance to distinguish between benign and malignant tumors. Open surgery by an expert surgeon with R0 target is the treatment of choice. Mitotane (alone or in combination with cytotoxic drugs) may be administered after surgery or in patients not amenable to surgery. The role of radiotherapy as an adjuvant treatment is uncertain whereas targeted radionuclide therapy seems to be a promising option. New adjuvant treatment options, even after complete tumor removal, are desired because postoperative disease-free survival at $5 \mathrm{yrs}$ is only around $30 \%$. The establishment of detailed guidelines with the purpose of optimizing therapy with only mitotane but also in combination with other antineoplasmatic drugs is still a task to be done. Future advances in the management of ACC will probably be connected with better understanding of the molecular pathogenesis (Adv Clin Exp Med 2015, 24, 2, 185-193).
\end{abstract}

Key words: molecular genetics, adrenocortical carcinoma, mitotane, treatment options.

\section{Epidemiology}

Adrenocortical tumors are quite common (prevalence of at least $3 \%$ in the population over the age of $50 \mathrm{yrs}$ ) whereas adrenocortical carcinoma (ACC) is a rare malignancy (incidence $1-2$ per $1 \mathrm{~m}$ population) with poor prognosis. Women are more often affected than man (ratio $1: 5$ ) [1]. Data concerning the epidemiology of ACC in Poland was not found. The age distribution model is bimodal, the disease is most commonly detected in the $5^{\text {th }}$ decade although there is a second peak in children under $10 \mathrm{yrs}$. Both groups differ in clinical presentation. In adults, $40 \%$ concern a nonsecretory mass detected incidentally or during evaluation for abdominal pain (median tumor size at diagnosis $>10 \mathrm{~cm}$ ). Only $60 \%$ of tumors present with hormone excess and the most common secretory syndrome is mixed Cushing's syndrome and virilization (35\%), followed by pure Cushing's syndrome $(30 \%)$ and pure virilization (20\%). Estrogen secreting tumors are rare (10\%) and the rarest are aldosterone-secreting ACCs [2]. In many patients with a seemingly hormonally inactive ACC, high concentrations of steroid precursors can be detected [1]. In contrast, in childhood, $90 \%$ of ACC present with hormone excess, the most common of which is androgen secretion (in 55\% of cases as the sole hormone or in combination with cortisol in approximately $30 \%$ of cases). Pure Cushing's syndrome is observed in less than 5\% of pediatric ACC cases [2]. An exceptionally high annual incidence of ACC in children has been described in southern Brazil (3.4-4.2 per $1 \mathrm{~m}$ children vs estimated worldwide incidence of 0.3 per $1 \mathrm{~m}$ children younger than $15 \mathrm{yrs}$ ). These cases are related to a TP53 tumor suppressor gene mutation [1].

\section{Molecular Genetics}

\section{Clonality and DNA Content}

Clonal analysis of tumors plays an important role in determining the cellular origin of the neoplasm and to uncover the fundamental mechanism 
of tumor progression. Monoclonality shows that tumor progression is initiated by internal genetic alterations, while polyclonality indicates that local or systemic stimuli influence tumor cells. $\mathrm{X}$-chromosome inactivation analysis has shown that ACCs initiate from a monoclonal population of cells, while ACAs might be both monoclonal and polyclonal [3]. Molecular techniques such as comparative genomic hybridization $(\mathrm{CGH})$ have revealed aneuploidy (a genomic aberration mostly observed in cancers) in 4 of 4 ACCs, whereas exclusively diploidy or tetraploidy are in normal adrenal cortices and ACAs. Further investigations have not shown any significant difference in overall survival among patients with ACC presenting aneuploidy and those with ACC presenting diploid neoplasm [4].

\section{Chromosomal Aberrations}

Comparative genomic hybridization (CGH) can be used to identify structural chromosomal alterations in ACCs. A large number of investigations have revealed ACAs have few regions of changes, whereas ACCs demonstrated complex chromosomal mutations. Most of the changes in ACCs concern gains in regions 4q, 4p16, 5p15, $5 q 12-13,5 q 32$-qter, 9q34, 12q13, 12q24, and 19p, and chromosomal losses were observed at $1 \mathrm{p}, 2 \mathrm{q}$, 11q 17p, 22p, and 22q [4]. Microsatellite examinations identified a high percentage of loss of heterozygosity $(\mathrm{LOH})$ and allelic imbalance at $17 \mathrm{p} 13$, $11 \mathrm{q} 15$ and 2 p16 $[4,5]$. The most recent study of 138 adrenal neoplasms demonstrated a higher number of alterations in ACCs (44\%) compared to ACAs (10\%) [4].

All these studies confirm the genetic variety and heterogeneity of chromosomal changes in ACCs, and the frequently-observed mutations at chromosomes 5, 12 and 17 are considered to play a fundamental role in tumorigenesis [4]. Moreover, CGH data has confirmed the theory of an adenoma-to-carcinoma progression according to more frequent chromosomal alterations in ACCs than ACAs and a positive correlation between the number of these changes and increasing tumor size [6].

\section{Gene Expression Profiles}

Global gene expression studies have identified that ACAs and ACCs have different expression profiles. An initial study reported high expression levels of genes involved in growth factor signaling and cell proliferation in ACCs (the so-called
IGF2 cluster), compared to the high expression levels of steroidogenic genes in ACAs (the steroidogenic cluster) [7]. Another study of 22 ACAs and 33 ACCs confirmed a significant difference in their expression profiles and found unique transcriptionally activated $(12 \mathrm{q}$ and $5 \mathrm{q})$ and repressed (11q,1p and 17p) chromosomal regions [4]. Recent studies have correlated expression profiles in ACC with clinical outcome and demonstrated that tumors with high histological grade were transcriptionally different from low grade tumors and presented decreased overall survival. A cluster analysis of ACCs revealed 2 different groups with distinct transcriptional signatures and clearly different clinical outcomes [7]. As a result, the poor outcome group had more genes involved in proliferation and the mitotic cell cycle, while the better outcome group presented an over-expression of genes implicated in differentiation, intracellular transport and metabolism [4].

\section{Gene Mutations}

Targeted genetic studies have shown that $\mathrm{LOH}$ of $17 \mathrm{p} 13,11 \mathrm{p} 15,11 \mathrm{q} 13,17 \mathrm{q} 22-24$ and $2 \mathrm{p} 16$ is more common in sporadic ACCs than in adrenocortical adenomas, while $\mathrm{CGH}$ analyses have shown that more number copy changes were present in ACCs than in ACAs [8]. Furthermore, a positive correlation was observed between the increasing number of genetic changes and increasing tumor size $[6,8]$.

\section{Epigenetics of Adrenocortical Tumors}

\section{DNA Methylation}

Changes at the epigenetic level have been inflected in carcinogenesis and considered diagnostic markers [4]. DNA methylation, which influences a number of various cellular processes responsible for apoptosis, cell cycle, DNA damage repair, growth factor response, signal transduction and tumor architecture, can bring on tumorigenesis and its progression [8]. This regulatory mechanism is frequently affected in cancer.

Fonesca studied the DNA methylation levels in 6 normal adrenal cortices, 15 ACCs, 27 ACAs and identified that tumor suppressor genes, responsible for cell cycle regulation and apoptosis, such as CDKN2A, GATA4, DLEC1, HDAC10, PYCARD and SCGB3A1, are relevantly hypermethylated in ACCs. Moreover, they found an inverse correlation between the levels of methylation and mRNA expression [7]. 
A recent investigation of 51 ACCs and 84 ACAs observed a correlation between the hypermethylation of promoters in ACCs and poor survival, and described H19, GOS2, PLAGLI and NDRG2 as silenced genes. Furthermore, it also provided insight into the possible function of methylation in ACC tumorigenesis, especially in the 11 p15 locus comprising IGF2 and H19 [4].

\section{MicroRNAs}

MicroRNAs are conserved small, noncoding RNAs implicated in the epigenetic regulation of cellular processes such as proliferation, apoptosis and differentiation. Alterations of miRNA including overexpression or deletion may be associated with cancer development and progression $[4,8]$.

The first examination of 36 adrenocortical samples (10 hormonally inactive ACAs, 9 cortisol producing ACAs, 10 normal cortical tissues and 7 ACCs) identified differential expression of 22 miRNAs, with 14 miRNAs preferentially expressed in ACCs. Including miR-184, miR-210, and miR-503 were up-regulated in ACCs, whereas miR-214, miR-375 and miR-511 were down-regulated [4]. Assessing levels of miR-184, miR-503 and miR-511 alone helped to distinguish between ACCs and ACAs. [4] Another miRNA analysis of adrenal samples (6 normal tissues, 22 ACAs, and 27 ACCs) revealed that 23 miRNA were differentially expressed between ACCs and ACAs, of these 14 up-regulated miRNAs and 9 down-regulated miRNAs uniquely to ACC. [4] Furthermore, the study reported a significant up-regulation of miR-483 (diagnostic sensitivity of $80 \%$ and specificity of $100 \%)$ and down-regulation of miR-195 and miR-335 in ACC. [4] In addition, miR-483 is located exactly within the IGF2 locus and it is supposed that dysregulation of the IGF2 locus affects the expression of miR-483 [7].

All these studies have confirmed that miRNAs may serve as promising biomarkers in ACCs due to their stability and the sensitivity of the detection methods available [8].

\section{Signaling Pathways}

\section{IGF Pathway}

Significant over-expression of IGF2 and downregulation of CDKN1C and $\mathrm{H} 19$ locus is observed in sporadic ACC [9]. The IGF2 gene is located on $11 \mathrm{p} 15$, which is organized into two different clusters: a telomeric domain consisting of IGF2 and H19 genes and a centromeric domain including the CDKN1C, KCNQ1OT1 and KCNQ1 genes [5]. The $11 \mathrm{p} 15$ region is subject to parental imprinting, in which specific genes are expressed solely either from the maternal or paternal allele [6].

IGF2 is maternally imprinted and is consequently expressed only from the paternal allele, whereas H19 and CDKN1C genes are both paternally imprinted and are therefore expressed from the maternal allele only. $[4,5]$ It has been reported that IGF2 over-expression is initiated by somatic structural alterations of the $11 \mathrm{p} 15$ locus, such as the loss of maternal imprinting or the loss of heterozygosity (two paternal alleles) [7]. The loss of heterozygosity at the $11 \mathrm{p} 15$ region is associated with poor outcome and appears more frequently in ACCs than in ACAs [9]. IGF2 regulates growth and apoptosis by connection with the insulin-like growth factor 1 receptor (IGF1R), which has also been shown to be over-expressed in ACCs, particularly in pediatric cases.

IGF2 genetic alterations of imprinted domains of the $11 \mathrm{p} 15$ region are implicated in the pathogenesis of Beckwith-Wiedemann Syndrome [4, 5]. This congenital overgrowth syndrome is characterized by macrosomia, macroglossia, organomegaly, developmental abnormalities and childhood tumors, which include ACC, nephroblastoma, hepatoblastoma, rhabdomyosarcoma.

The fact of IGF2 over-expression and the high incidence of ACC in BWS make the IGF system an interesting target for pharmacological inhibition [4].

\section{WNT Activation}

The WNT/ $\beta$-catenin signaling pathway is a principal developmental pathway in multiple organ systems, including the adrenal gland and normally activated during embryonic development [4].

$\beta$-catenin plays the main role in this signaling pathway, participating in cell-cell adhesion, as a transcription cofactor with T-cell factor/lymphoid enhancer factor mediating transcription activation of target genes of the Wnt signaling pathway [5].

Genetic mutations of the $\mathrm{WNT} / \beta$-catenin system were initially identified in familial adenomatous polyposis (FAP). Recent studies of adrenocortical tumors assume that the $\mathrm{WNT} / \beta$-catenin signaling pathway plays a crucial role in sporadic adrenocortical tumorigenesis [4].

Furthermore, gene expression profiling analysis disclosed over-expression of $\beta$-catenin target genes, implying an importance of active $\beta$-catenin signaling in ACCs. $\beta$-catenin alteration was observed in both ACAs and ACCs, which indicates 
that WNT activation may be an early step in adrenocortical tumorigenesis, which preludes malignant transformation [4].

\section{Pathogenesis}

The role of pathologist is to diagnose an adrenocortical tumor, to differentiate a malignant from a benign tumor and to assess its prognosis [10]. Thus, special diagnostic algorithms have been developed to combine a variety of clinical, histological and immunohistochemical parameters [4]. The first step is macroscopic examination. Most ACCs weigh more than $100 \mathrm{~g}$ and are generally larger than $5-6 \mathrm{~cm}$ in diameter, while benign adrenocortical tumors weigh less than $50 \mathrm{~g}$ and are usually smaller than 5-6 cm. Malignant tumors are often lobulated [10].

A combination of histological parameters is helpful to identify tumors with malignant potential and allow the calculation of a 'score' for a given tumor $[5,10]$. The Weiss system appears to be the most widely used classification, composed of 9 different items [5]. It is assumed that a score above 3 determines malignant tumors, while tumors without these features are less likely to metastasize and are considered benign $[4,5]$. As mentioned previously, IGF2 and allelic losses at 17p13 have been considered promising markers [5]. Immunohistochemical criteria indicate the importance of Ki-67 and cyclin E, which may be useful as diagnostic markers for malignancy in adrenocortical tumors $[5,10]$.

The histopathological criteria proposed by Weiss (Weiss, 1984) for establishing a differential diagnosis between ACC and adenoma are shown in Table 1. A score of 3 or more correlates with malignancy.

\section{Prognosis}

A combination of various criteria including clinical, biochemical, macroscopic, histological, immunohistochemical and molecular help to assess the prognosis of ACCs [10]. Among the clinical parameters, the most commonly used is McFarlane staging (Table 2). A prognosis of stages 1 and 2 tumors is better than that of stages 3 or 4 tumors [5].

On the pathological level, tumor size, mitotic count, Ki-67 and cyclin E have been suggested to associate with shorter survival [10]. Tumors larger than $12 \mathrm{~cm}$ confer a worse prognosis as well as high tumor grade (> 20 mitoses per HPF) and velocity of tumor growth $[4,5]$.
Table 1. Weiss criteria for malignancy [10]

\begin{tabular}{|l|l|}
\hline Histological criteria & Weighted value (0 or 1) \\
\hline High nuclear grade & $\begin{array}{l}\text { and } 2 \\
0\end{array}$ \\
\hline Mitoses & $\begin{array}{l}\leq 5 \text { per } 50 \mathrm{HPF} \\
0\end{array}$ \\
\hline Abnormal mitosis & $\begin{array}{l}\text { absent } \\
0\end{array}$ \\
\hline Clear cells & $>25 \%$ \\
& 0 \\
\hline Diffuse architecture & $\leq 33 \%$ surface \\
& 0 \\
\hline Necrosis & absent \\
& 0 \\
\hline Venous invasion (smooth & absent \\
muscle in wall) & 0 \\
\hline Sinusoidal invasion & absent \\
\hline Capsular invasion & 0 \\
\hline & absent \\
\hline
\end{tabular}

HPF - high power fields.

Table 2. McFarlane classification [10]

\begin{tabular}{|l|l|}
\hline Stage I & tumor $\leq 5 \mathrm{~cm}$ \\
\hline Stage II & tumor $>5 \mathrm{~cm}$ \\
\hline Stage III & $\begin{array}{l}\text { any tumor size and mobile nodes or } \\
\text { Infiltration locally reaching neighboring } \\
\text { organs and no lymph node }\end{array}$ \\
\hline Stage IV & $\begin{array}{l}\text { invasion of neighboring nodes or any } \\
\text { tumor size and fixed nodes or any tumor } \\
\text { size, any lymph nodes and metastasis }\end{array}$ \\
\hline
\end{tabular}

A better survival is usually associated with younger patients [5]. A cortisol secreting tumor is reported to be an adverse prognostic factor [5].

\section{Imaging}

The most common techniques (CT and MRI) and recently also FDG-PET use both size and appearance to distinguish between benign and malignant tumors. The size of an adrenal tumor remains one of the best indicators of malignancy [1]. Current guidelines recommend surgical removal of tumors greater than $5 \mathrm{~cm}$ (though in the post-surgical examination $75 \%$ of them turn out to be benign) [2]. Measurement of Hounsfield units (HU) in unenhanced CT is of great value in 
differentiating malignant from benign adrenal lesions. If the lesion density is high (above $20 \mathrm{HU}$ ), a malignant tumor or pheochromocytoma should be suspected. For a better discrimination of lipid-poor adenomas from ACC, a delayed contrast-enhanced CT can be used, analyzing washout of a contrast medium. Modern MRI with dynamic gadolinium enhanced and chemical shift technique is equally effective as CT in distinguishing malignant from benign tumors by assessment of fat content. This technique enables better assessment of invasion into adjacent organs and the inferior vena cava, which is useful in planning surgery. Adrenal scintigraphy with iodocholesterol analogs is not widely used as it is time-consuming and is associated with a relatively high dosage of radiation. In contrast, recent studies have demonstrated good performance of FDG-PET in differentiating malignant from benign adrenal lesions in patients with proven or suspected malignancy. A new method for adrenal imaging is ${ }^{11} \mathrm{C}$-metomidate-PET [1]. Metomidate binds both to adrenal $11 \beta$-hydroxylase and aldosterone synthase with high specificity and affinity and is therefore an excellent tool to distinguish lesions of adrenocortical origin from other lesions (high tracer uptake in both primary tumor and metastases) [11].

\section{Hormonal Assessment}

Some hormone secretion patterns suggest the malignant potential of the evaluated tumor (e.g. estradiol in males, high concentrations of DHEA-S or secretion of steroid precursors). Hormone measurements enable proper preparation for surgical procedures (e.g. risk of postoperative adrenal insufficiency connected with autonomous cortisol secretion by the tumor) and they are also essential

Table 3. Hormonal work-up and imaging in patients with suspected or proven ACC (recommendation of the ACC working group of the European Network for the Study of Adrenal Tumors, May 2005) [1]

\begin{tabular}{|l|}
\hline Hormonal work-up \\
\hline Glucocorticoid excess (min 3 of 4 tests) \\
\hline Sexual steroids and steroid precursors \\
\hline Mineralocorticoid excess \\
\hline Exclusion of a pheochromocytoma (1 of 2 tests) \\
\hline Imaging \\
\hline $\begin{array}{l}\text { CT or MRI of abdomen and thorax } \\
\text { Bone scintigraphy (when suspecting skeletal metastases) } \\
\text { FDG-PET (optional) }\end{array}$ \\
\hline
\end{tabular}

for the establishment of tumor markers for monitoring of tumor recurrence [1].

\section{Staging}

Until 2004, no official TNM classification was available for ACC and different staging systems were used, most often the Sullivan modification of the McFarlane system. According to the TNM staging system for ACC proposed by the International Union Against Cancer in 2004, stages I and II describe localized tumors up to $5 \mathrm{~cm}$ and larger than $5 \mathrm{~cm}$, respectively. Locally invasive tumors or the presence of local lymph nodes are classified as stage III, whereas stage IV consists of tumors invading adjacent organs or presenting with distant metastases. The main purpose of the staging system is to predict the disease-free and disease-specific survival in patients with cancer. For many tumors, the TNM system has been modified to improve accuracy. On the basis of the analysis of the German ACC Registry, a revised classification has been proposed (the European Network for the Study of Adrenal Tumor classification). In this system, stage III ACC is defined by the presence of positive lymph nodes, infiltration of surrounding tissue or presence of tumor thrombus in the vena cava or renal vein (VTT), whereas stage IV is restricted to patients with distant metastases. There is also a suggestion that correct staging can be performed only after surgery and that the result of surgery should be taken into account (e.g. tumor spillage during surgery represents tumor spread and is connected with worse prognosis, so assignment of these cases to stage III or even IV irrespective of prior surgery staging may be justified) [12].

\section{Surgery}

In stage I-III, surgery is recommended and complete resection offers the best chance for cure, though adjuvant therapy may be also required. In stage IV, surgery is also taken under consideration - incomplete resection of the primary tumor or metastatic disease not amenable to surgery are associated with poor prognosis, however tumor debunking may help to control hormone excess and in some cases enable other therapeutic options [1]. Surgery for local recurrence or metastatic disease is accepted and is associated with improved survival in retrospectives studies $[1,13]$. The best predictors of prolonged survival after the first recurrence are time to first recurrence (TTFR) over 12 months and tumors amenable to radical resection. When both conditions are fulfilled, surgery is recommended [13]. 
At present, open surgery is regarded as the standard of care and the use of laparoscopic adrenalectomy is still a matter of debate $[1,13]$. However, according to Martin Fassnacht, the experience of the surgeon is more important than the technique of the procedure (ECE 2014 lecture: adrenocortical carcinoma - current concepts and future perspectives).

\section{Therapy}

Adjuvant treatment options even after complete tumor removal are desired because postoperative disease-free survival at 5 yrs is only around $30 \%$. At present, no such options have been convincingly established [1].

\section{Mitotane}

Mitotane (o,p-DDD) is the only adrenal-specific agent available for the treatment of ACC. The first description of its effects was made in 1948, and it concerned adrenal atrophy in dogs [14]. Despite the long history of mitotane use in ACC, it is not available in all countries. Mitotane has an impact (as a cytotoxic agent) on the fascicular and reticular zone, leading to their degeneration, whereas changes of the zona glomerulosa are relatively slight. Metabolic activation is essential for its adrenolytic activity. Oxidative damage through the production of free radicals may contribute to the adrenolytic effect of mitotane. Impairment of adrenal steroidogenesis is due to a direct inhibitory effect on steroidogenic enzymes (inhibition of 11 beta-hydroxylation) [1]. Mitotane is administered per os, and monitoring of blood levels is mandatory for predicting the efficacy and toxicity (therapeutic range $14-20 \mathrm{mg} / \mathrm{L}$ ). The daily dosage needed to achieve and maintain blood levels greater than $14 \mathrm{mg} / \mathrm{L}$ is variable. The response rate fluctuates in different publications. Allolio and Fassnacht have analyzed the efficacy of mitotane treatment in advanced ACC, including only prospective studies or reports with more than 10 patients from the last 20 yrs. Based on this analysis, it was concluded that mitotane leads to an objective tumor regression in about $25 \%$ of cases and control of hormone excess in the majority of patients. Although a complete response (or even cure) in patients with advanced ACC is extremely rare, long-term survival has been reported $[1,15]$. Mitotane has a narrow therapeutic window, and adverse effects occur frequently and are often dose limiting. More than $80 \%$ of all patients experience at least one undesirable effect [1]. These effects are mainly gastrointestinal or neurological. Abnormalities in laboratory tests like leucopenia, hypercholesterolemia, hypertriglyceridemia (very common), thrombocytopenia and anemia (common) may be observed. In general, the adverse effects are reversible after cessation of mitotane. The probability of central neurological system (CNS) adverse effects increases strongly with mitotane blood levels greater than $20 \mathrm{mg} / \mathrm{L}$ (above the therapeutic range) [1]. In case of overdose, cessation of administration is the only way to reduce drug levels. There is no antidote and the drug cannot be removed by hemodialysis, because it is lipophilic. Patients with renal or liver insufficiency, obesity or those who have lost weight recently are more exposed to overdose.

For management of nausea, 5-hydroxytryptamine blockers may be useful. In the case of significant neuropsychiatric side effects, drug treatment is interrupted for a minimum of 1 week and restarted with a lower dose. Treatment with mitotane, due to its adrenolytic activity, leads to adrenal insufficiency in the majority of patients and it also increases the metabolic clearance of glucocorticoids, and thus high-dose glucocorticoid replacement (e.g. $50 \mathrm{mg}$ hydrocortisone daily) is needed. Inadequate glucocorticoid substitution enhances the mitotane-induced adverse effects and reduces mitotane tolerance [1]. Mitotane is a therapeutic indication in the treatment of inoperable ACC and in preparing for adrenalectomy. However, the high rate of recurrence implies a need for adjuvant therapy following surgical procedures. In Poland, Kasperlik-Załuska described a group of 82 patients with microscopically confirmed adrenocortical carcinoma, who were treated with mitotane after surgical procedure irrespectively of stage at the time of surgery. In conclusion, immediate administration of mitotane after surgery was connected with better survival in comparison to the group with delayed treatment [16]. The topic was also discussed in 2008 by an international panel of experts. Adjuvant therapy with mitotane was unanimously recommended for patients with potential residual disease ( $\mathrm{R} 1$ or $\mathrm{Rx}$ resection) or greater than $10 \%$ Ki67 positivity on pathologic examination, whereas adjuvant therapy was not considered mandatory in patients fulfilling all of the following criteria: stage I or II (based on ENSAT TNM criteria), histologically proven $\mathrm{R} 0$ resection and Ki67 expression $\leq 10 \%$. Adjuvant therapy in stage III after R0 resection is still an issue to be resolved $[17,18]$. Currently, to evaluate the effectiveness of adjuvant therapy with mitotane, a randomized prospective study ADIUVO is ongoing (www.adiuvo-trial.org). Patients will be randomly assigned to receive mitotane treatment or observational 
follow-up only. Mitotane will be administered until progression or unacceptable toxicity for a minimum of 2 years. The administration of any other anticancer agents including chemotherapy and active biologic agents is not permitted. Two hundred patients (100 per treatment arm) will be enrolled. The duration of the study will be 6 years (enrollment period, 4 years; follow-up period, 2 years).

\section{Cytotoxic Chemotherapy}

The response rates are generally poor. The best results have been initially described for the so-called Italian protocol (Berutti et al.) which combines mitotane with etoposide, doxorubicin and cisplatin (EDP-M). According to WHO criteria, the overall response rate in 72 patients was $49 \%$, including 5 patients with complete response $[1,19]$. The second active regime described was a combination of mitotane and streptozocine (Khan et al.), and complete or partial responses were observed in $36 \%$ of patients $[1,20]$. The promising results of both protocols led to the first ever phase III trial in ACC directly comparing these treatment options [First International Randomized Trial in Locally Advanced and Metastatic Adrenocortical Carcinoma Treatment (FIRM-ACT)]. The publication of the results of FIRM-ACT (2012) showed an advantage to EDP-M in terms of progression-free survival. Based on this data, EDP-M can now be considered the "standard of care" though the $25 \%$ response rate (with another $28 \%$ disease stabilization) is less optimistic than data obtained in primary observations by Berutti et al. Additionally, the retrospective reviews of the effects of mitotane as a single agent place the response rate in a similar range [21]. The limited response to cytotoxic therapy in ACC is linked to high expression of the multidrug-resistant gene mdr- 1 , resulting in high concentrations of p-glycoprotein acting as a drug efflux pomp, transporting cytotoxic agents (e.g. doxorubicin) out of the cell. The inhibition of a drug efflux pomp could enhance the efficacy of cytotoxic chemotherapy, and though early trials were not promising, the search for more potent mdr-1 inhibitors is ongoing [1].

\section{Radiotherapy}

ACC has been considered radio resistant for a long time, however several reports have described tumor response rates up to $42 \%$, and it has also been demonstrated that radiotherapy reduces the risk of local failure.

Existing data regarding radiotherapy in ACC indicates that this treatment should be taken under consideration especially when microscopic tumor residues are detectable after surgery (R1) or residual tumor dimensions are not known (RX). Whereas macroscopic visible residual tumors (R2) are indication for a second operation [22]. Currently, there are no guidelines for radiotherapy in patients who have undergone complete tumor removal (R0), although this treatment is usually not recommended when tumor dimensions are not greater than $8 \mathrm{~cm}$. Radiotherapy may be considered as an additional option after R0 resection for tumors with greater dimensions, blood vessel invasion and Ki-67 index $\geq 10 \%$, which are associated with a high recurrence risk. Combined treatment (radiotherapy and cytotoxic drugs, such as mitotane) is also under investigation [22].

\section{Targeted Radionuclide Therapy}

Hahner et al. reported about the attempt of salvage treatment with $\left[{ }^{131} \mathrm{I}\right]$ iodometomidate. Between 2007 and 2010, $\left[{ }^{131} \mathrm{I}\right]$ iodometomidate was administered to 11 patients. One patient died 11 days after treatment, unrelated to the radionuclide therapy. The best response was classified as a partial response in one case, in 5 patients stable disease was observed. Treatment was generally well tolerated and transient bone marrow depression was observed. The study has important limitations due to the small number of treated patients. Radionuclide therapy is a promising treatment option deserving evaluation in prospective clinical trials [11].

\section{Future Treatments}

Over-expression of the epidermal growth factor receptor (EGFR) protein in AC has been considered a promising target for the use of EGFR inhibitors. Unfortunately, treatment with tyrosine kinase inhibitors revealed limited efficacy [23]. Better results have been noted with targeted therapy of insulin-like growth factor 1 receptor (IGF-1R) inhibitors. The IGF system is seen as an interesting aim for future therapies in advanced ACC and further clinical investigations are currently in progress [24]. Vascular endothelial growth factor (VEGF) has also been reported as an attractive therapeutic approach for ACC, however the results of studies did not meet expectations [23]. A further object of interest is $\mathrm{Wnt} / \beta$-catenin pathway and so far, its antagonists remain in preliminary preclinical investigations [8].

\section{Treatment of Hormone Excess}

Management of endocrine syndromes is often important because the associated systemic effects may significantly impact patient well-being. 
Treatment with mitotane leads to adrenal insufficiency in the majority of patients, though the onset is delayed, and some other drugs may be required to control hormone excess at the beginning of treatment. Amiloride can be used to correct hypokalemia. Based on a case report, the use of spironolactone may impair the antitumor activity of mitotane [1]. Adrenostatic drugs like ketoconazole, metyrapone, aminoglutethimide and etomidate can be used to lower cortisol into the normal range. Ketoconazole (400-1200 mg/d) is most often used though the treatment may be connected with serious hepatotoxicity. Metyrapone treatment is limited by poor tolerance. Common side-effects include nausea, vomiting, abdominal discomfort, headache, dizziness and sedation. Hypotension may be also observed. Intravenous etomidate (e.g. $80 \mathrm{mg} / \mathrm{d}$ as a continuous infusion) can be used in emergencies (e.g. glucocorticoid-induced psychosis) but is not suitable for long-term treatment due to administration only by intravenous route [1]. Mifepristone may be another therapeutic option. It is well known for its antiprogestin activity but it is also a glucocorticoid receptor antagonist with rapid onset of action and therefore can be used to treat hypercortisolism. The mechanism of action may lead to severe hypokalemia and hypertension, as mifepristone blocks only glucocorticoid action (ACTH and cortisol levels remain elevated) whereas the mineralocorticoid activity of cortisol excess is not affected by mifepristone. The assessment of efficacy of the therapy and risk of signs of adrenal insufficiency during treatment is difficult because it is only based on clinical features [25].

\section{Conclusion}

Adrenocortical carcinomas (ACCs) are rare, aggressive tumors with dismal prognosis. Considering the rarity and complexity of ACC, significant progress has been made over the past decade to understand its molecular pathogenesis, better diagnosis and better prediction of prognosis. These advances help to improve the clinical care of patients with ACC and hopefully will also give new perspectives for possible targeted therapies.

\section{References}

[1] Allolio B, Fassnacht M: Adrenocortical Carcinoma: Clinical Update. J Clin Endocrinol Metab 2006, 91, $2027-2037$.

[2] Kirschner LS: Emerging Treatment Strategies for Adrenocortical Carcinoma: A New Hope. J Clin Endocrinol Metab 2006, 91, 14-21.

[3] Libé R, Fratticci A, Bertherat J: Adrenocortical cancer: pathophysiology and clinical management. Endocr Rel Cancer 2007, 14 13-28.

[4] Else T, Kim A, Sabolch A, Raymond VM, Kandathil A, Caoili EM, Jolly S, Miller BS, Giordano TS, Hammer GD: Adrenocortical Carcinoma. Endocr Rev 2014, 35, 282-326.

[5] Libé R, Gicquel C, Bertagna X, Bertherat J: Molecular genetics of adrenal cortical carcinoma. In: Adrenal Cancer, Bertagna X, John Libbey Eurotext, Montrouge 2006, $1^{\text {st }}$ ed., 13-24.

[6] Soon PSH, McDonald KL, Robinson BG, Sidhu SB: Molecular Markers and the Pathogenesis of Adrenocortical Cancer. Oncologist 2008, 13, 548-561.

[7] Lerario A, Moraitis A, Hammer G: Genetics and epigenetics of adrenocortical tumors. Mol Cell Endocrinol 2014, $386,67-84$.

[8] Xu Y, Qi Y, Zhu Y, Ning G, Huang Y: Molecular markers and targeted therapies for adrenocortical carcinoma. Clin Endocrinol 2014, 80, 159-168.

[9] Lehmann T, Wrzesiński T: The molecular basis of adrenocortical cancer. Cancer Genetics 2012, 205, 131-137.

[10] Tissier F: Pathological pattern of adrenal cortical carcinoma. In: Adrenal Cancer, Bertagna X, John Libbey Eurotext, Montrouge 2006, $1^{\text {st }}$ ed., 25-44.

[11] Hahner S, Kreissl MC, Fassnacht M, Haenscheid H, Knoedler P, Lang K, Buck AK, Reiners C, Allolio B, Schirbel A: [131I] Iodometomidate for Targeted Radionuclide Therapy of Advanced Adrenocortical Carcinoma. Clin Endocrinol Metab 2012, 97, 914-922.

[12] Fassnacht M, Johanssen S, Quinkler M, Bucsky P, Willenberg HS, Beuschlein F, Terzolo M, Mueller HH, Hahner S, Allolio B: Limited Prognostic Value of the 2004 International Union Against Cancer Staging Classification for Adrenocortical Carcinoma Proposal for a Revised TNM Classification. Cancer 2009, 115, 243-250.

[13] Erdogan I, Deutschbein T, Jurowich C, Kroiss M, Ronchi C, Quinkler M, Waldmann J, Willenberg HS, Beuschlein F, Fottner C, Klose S, Heidemeier A, Brix D, Fenske W, Hahner S, Reibetanz J, Allolio B, Fassnacht M: The Role of Surgery in the Management of Recurrent Adrenocortical Carcinoma. J Clin Endocrinol Metab 2013, 98, 181-119.

[14] Stigliano A, Cerquetti L, Sampaoli C, Bucci B, Toscano V: Current and Emerging Therapeutic Options in Adrenocortical Cancer Treatment. J Oncol 2012, Article ID 408131, 13 pages. doi:10.1155/2012/408131.

[15] Bednarek-Tupikowska G, Florczak A, Witkiewicz W, Tupikowski W, Cisarz E: A long term survival of the patient treated due to advanced adrenocortical carcinoma. Pol Arch Med Wewn 2005, 113, 462-465.

[16] Kasperlik-Załuska AA: Clinical results of the use of mitotane for adrenocortical carcinoma. Braz J Med Biol Res 2000, 33, 1191-1196. 
[17] Berruti A, Fassnacht M, Baudin E, Hammer G, Haak H, Leboulleux S, Skogseid B, Allolio B, Terzolo M: Adjuvant Therapy in Patients With Adrenocortical Carcinoma: A Position of an International Panel. J Clin Oncol 2010, 28, 401-402.

[18] Lebastchi AH, Kunstman JW, Carling T: Adrenocortical Carcinoma: Current Therapeutic State-of-the-Art. J Oncol 2012, Article ID 234726, 11 pages, 2012. doi:10.1155/2012/234726.

[19] Berruti A, Terzolo M, Sperone P, Pia A, Della Casa S, Gross DJ, Carnaghi C, Casali P, Porpiglia F, Mantero F, Reimondo G, Angeli A, Dogliotti L: Etoposide, doxorubicin and cisplatin plus mitotane in the treatment of advanced adrenocortical carcinoma: a large prospective phase II trial. Endocr Rel Cancer 2005, 12, 657-666.

[20] Khan TS, Imam H, Juhlin C, Skogseid B, Gröndal S, Tibblin S, Wilander E, Öberg K, Eriksson B: Streptozocin and o,p'DDD in the treatment of adrenocortical cancer patients: Long-term survival in its adjuvant use. Ann Oncol 2000, 11, 1281-1287.

[21] Kirschner LS: Gaining Traction in the Treatment of Adrenocortical Carcinoma. J Clin Endocrinol Metab 2013, 98, 45-47.

[22] Polat B, Fassnacht M, Pfreundner L, Guckenberger M, Bratengeier K, Johanssen S, Kenn W, Hahner S, Allolio B, Flentje M: Radiotherapy in Adrenocortical Carcinoma. Cancer 2009, 115, 2816-2823.

[23] Glover AR, Ip J, Ting Zhao J, Soon PSH, Robinson BG, Sidhu SB: Current management options for recurrent adrenocortical carcinoma. Onco Targets Ther 2013, 6, 635-643.

[24] Tacon LJ, Prichard RS, Soon PSH, Robinson BG, Clifton-Bligh RJ, Sidhu SB: Current and Emerging Therapies for Advanced Adrenocortical Carcinoma. Oncologist 2011, 16, 36-48.

[25] Castinetti F, Fassnacht M, Johanssen S, Terzolo M, Bouchard P, Chanson P, Do Cao C, Morange I, Picó A, Ouzounian S, Young J, Hahner S, Brue T, Allolio B, Conte-Devolx B: Merits and pitfalls of mifepristone in Cushing's syndrome. Eur J Endocrinol 2009, 160, 1003-1010.

\section{Address for correspondence:}

Grażyna Bednarek-Tupikowska

Department of Endocrinology, Diabetology and Isotope Therapy

Wroclaw Medical University

Pasteura 4

50-367 Wrocław

Poland

Tel.: +48717842549

E-mail: grazyna.bednarek-tupikowska@umed.wroc.pl

Conflict of interest: None declared

Received: 10.08 .2014

Revised: 26.08.2014

Accepted: 17.10.2014 